\title{
Como a interação universidade-empresa é remunerada no Brasil: evidências dos grupos de pesquisa do CNPq
}

\author{
Márcia Siqueira Rapini* \\ Vanessa Parreiras de Oliveira** \\ Thiago Caliari***
}

Recebido: 31/03/2015 Versão revisada (entregue): 04/01/2016 Aprovado: 06/01/2016

\section{RESUMO}

O objetivo deste artigo é investigar o financiamento da interação universidade-empresa (UE) no Brasil. Foram analisados os tipos de relacionamento e suas respectivas remunerações referentes a 2.726 grupos de pesquisa do Censo 2008 do Diretório de Grupos de Pesquisa/ CNPq. A análise aponta que os tipos de remuneração mais frequentes foram a transferência de recursos financeiros e materiais entre as partes e, em menor proporção, as remunerações vinculadas à geração e à troca de conhecimento por meio de bolsas e intercâmbio de pessoal. Usando um modelo econométrico logit multinomial, foram analisadas características que influenciam no tipo de remuneração utilizado na interação U-E. Os resultados sugerem que a excelência científica, a grande área de conhecimento, o modo de interação e o setor de atuação da empresa impactam de maneira diferenciada no tipo de remuneração estabelecido.

PALAVRAS-CHAVE | Financiamento; Remuneração; Interação Universidade-Empresa; Grupos de Pesquisa do CNPq; Brasil

\section{Código-JEL | O30}

* Universidade Federal de Minas Gerais (UFMG), Belo Horizonte (MG), Brasil. E-mail: msrapini@cedeplar.ufmg.br

** Universidade Estadual de Campinas (Unicamp), Campinas (SP), Brasil. E-mail: vparreiras@uol.com.br

*** Universidade Federal de Alfenas (Unifal), Alfenas (MG), Brasil. E-mail: thiago.caliari@unifal-mg.edu.br 


\section{How university-firms interaction is remunerated in Brazil: evidences from CNPq research groups}

\section{ABSTRACT}

The aim of this paper is to investigate the funding of university-industry interactions in Brazil. The responses of 2,726 interactive research groups from Diretório de Grupos de Pesquisa/ $\mathrm{CNPq}$ relating to Census 2008 were analyzed. The analysis shows that most frequent remuneration was financial and material resources and also, to a lesser extent, the ones related to knowledge generation and exchange. Using a multinomial logit econometric model, the characteristics that influence the type of remuneration in university-firm cooperation were analyzed. The results suggest that scientific excellence, the research group knowledge area, the mode of interaction and the industrial sector impact differently on the established remuneration type.

KEYWORDS | Funding; Remuneration; University-Firms Interactions; CNPq Research Groups; Brazil.

JEL CODE | O30 


\section{Introdução}

A literatura sobre sistemas nacionais de inovação (SNI) atribui importância decisiva à interação entre produção científica e tecnológica (LUNDVALL, 1992; NELSON, 1993; FREEMAN, 1995). Segundo a teoria, as universidades e os institutos públicos de pesquisa (IPP), principais atores envolvidos na geração de ciência básica e aplicada, são partes importantes do SNI, uma vez que contribuem direta e indiretamente para o processo de inovação nas empresas. Esta contribuição abrange fontes de conhecimentos geral e específico, desenvolvimento de novos instrumentos e técnicas, treinamentos de pessoal e criação de empresas spin-offs.

Dentre as razões atribuídas à aproximação das universidades com o setor produtivo, identificam-se, do lado das empresas (MOWERY; SAMPAT, 2005): custo crescente da pesquisa associada ao desenvolvimento de produtos e serviços para assegurar posiçôes vantajosas no mercado; necessidade de compartilhar o custo e o risco das pesquisas pré-competitivas com outras instituiçôes que dispõem de suporte financeiro governamental; elevado ritmo de introdução de inovações no setor produtivo e redução do intervalo de tempo que decorre entre a obtenção dos primeiros resultados de pesquisa e sua aplicação; e decréscimo dos recursos governamentais para pesquisa em setores antes fortemente fomentados, como os relacionados ao complexo industrial militar. Do lado da universidade, as motivaçôes principais seriam: dificuldade crescente para obtenção de recursos públicos para a pesquisa universitária; e interesse da comunidade acadêmica em legitimar seu trabalho junto à sociedade que é, em grande medida, a responsável pela manutenção das instituições universitárias.

No Brasil, o debate sobre a interação universidade-empresa (U-E) remonta ao final da década de 1970, com o esgotamento do modelo de substituição de importações e a necessidade de construir capacidades científicas nacionais. Entretanto, só recentemente as interações U-E vêm sendo estimuladas de forma sistemática pela política de ciência, tecnologia e inovação (C\&T\&I). Inúmeros instrumentos vêm sendo criados, abrangendo desde o incentivo à pesquisa cooperada, a inserção de pesquisadores nas empresas, podendo os recursos serem alocados nas universidades e/ou nas empresas (subvenção), até a criação de institucionalidades que favoreçam a inovação - como incubadoras e parques tecnológicos - e a aproximação U-E - com os Núcleos de Inovação Tecnológica (NIT).

Apesar de as contribuições de universidades e IPPs para o processo de inovação nas empresas serem relevantes, a literatura que investiga a interação universidade/ 
IPP-empresa sofre de importantes limitaçôes no que tange aos esforços empíricos para examinar o financiamento dos processos cooperativos entre estas instituições. Buscando avançar nesta temática, o objetivo do presente artigo é investigar como as interaçōes U-E são remuneradas no Brasil. A hipótese é a de que as interações U-E no país seguem padrōes variados de remuneração que dependem de características intrínsecas aos grupos, empresas e tipos de interação.

Para tanto, utilizou-se um conjunto de informaçōes do Diretório dos Grupos de Pesquisa no Brasil, do Conselho Nacional de Desenvolvimento Científico e Tecnológico (DGP/CNPq), até agora pouco explorado: a remuneração dos relacionamentos entre os grupos de pesquisa (GPs) e o setor produtivo. O universo investigado compõe-se de 2.726 GPs de universidades/IPPs brasileiros que interagiram com o setor produtivo no Censo 2008.

O texto está organizado em seis seções, além desta introdução. A seguir é feita uma breve revisão sobre o financiamento da interação U-E, discorrendo mais sobre sua vertente relacionada a diretrizes legais, instrumentos e programas de apoio à interação U-E no Brasil. Posteriormente, apresentam-se a base de dados e a metodologia. Também são mostrados a distribuição dos GPs nas grandes áreas do conhecimento, os modos de interação com o setor produtivo e os tipos de remuneração. Discutem-se os resultados do modelo logit multinomial proposto para analisar características que influenciam no tipo da remuneração utilizada na interação U-E e, por fim, são tecidas as considerações finais acerca do trabalho.

\section{0 financiamento da interação universidade-empresa}

Os artigos seminais de Arrow ([1962] 2002) e Nelson ([1959] 2002) apresentam as razóes econômicas para o financiamento público da pesquisa científica. A pesquisa básica possui algumas características como incerteza, difícil apropriabilidade e indivisibilidade, que geram um subinvestimento por parte de agentes privados. Isso justificaria, pois, o financiamento público. Como o progresso técnico é considerado elemento importante para o desenvolvimento econômico, promovê-lo tornou-se um raciocínio articulado de apoio público à pesquisa universitária.

De acordo com Nelson (1990), as firmas financiam a pesquisa universitária com a finalidade de auferir algum tipo de vantagem de acesso àquela pesquisa ou aos seus resultados. Desse modo, quando as empresas financiam a pesquisa acadêmica, espera-se que estas afetem não somente os resultados, mas também 
os benefícios desta ação. Em uma perspectiva econômica evolucionária, a busca pela diferenciação e inovação conduz ao mecanismo de concorrência no mercado, sendo que a cooperação é um ingrediente vital nos sistemas adaptativos complexos (BEINHOCKER, 2007), de modo que as empresas são incentivadas a buscar por interaçôes com universidades.

Ainda que não seja fomentada pela lógica do mercado, a pesquisa universitária vem aumentando sua contribuição para as atividades inovadoras das empresas. As universidades não são mais "torres de marfim" destinadas à produção de conhecimento para seu próprio uso, tornando-se instrumentos de uma economia baseada no conhecimento para promover o desenvolvimento e a mudança (MOWERY; SAMPAT, 2005).

Ademais, as diversas mudanças na década de 1980 (crises econômicas e elevações inflacionárias, que colocaram os orçamentos nacionais sob pressão) vêm impondo pressões crescentes sobre o financiamento da pesquisa pública universitária. Esta nova base lógica para alocação de recursos foi fortalecida pelas pressões para que a política científica e de inovação "faça a pesquisa científica mais relevante do que no passado" (GOLDFARB, 2008, p. 43). Desse modo, as universidades vêm sendo incentivadas a contribuir mais diretamente para a inovação industrial e o crescimento econômico (local) e encorajadas a se engajarem na comercialização do conhecimento (REDDY, 2011).

É exatamente neste contexto que o debate acerca do financiamento à interação U-E ganha destaque na literatura, sendo pautado em duas principais agendas de pesquisa. Uma procura identificar os possíveis impactos do aumento do financiamento privado nas atividades acadêmicas, analisado no desempenho das publicações (GULBRANDSEN; SMEBY, 2005; PERKMANN; WALSH, 2009; VAN LOOY et. al., 2004) e na agenda de pesquisa (FLORIDA; COHEN, 1999; VAN LOOY et. al., 2004). A outra analisa os instrumentos de política que vêm sendo criados para fomentar tal articulação e os incentivos econômicos propiciados pelos mesmos.

$\mathrm{Na}$ maior parte dos casos, as políticas de inovação incluem o desenvolvimento e a difusão de novas tecnologias por meio da promoção das atividades de P\&D e do estímulo à difusão e cooperação nas áreas de pesquisa genérica de longo prazo. Além de projetos de $\mathrm{P} \& \mathrm{D}$ conjuntos, tais ações têm incluído a formação e a capacitação de recursos humanos (RHs), informação, design, etc. (CASSIOLATO; LASTRES, 2005). A próxima seção analisa os instrumentos de apoio à interação U-E no Brasil. 


\subsection{Os instrumentos de apoio à interação universidade-empresa no Brasil no período recente}

No Brasil, os mecanismos de financiamento público priorizaram, desde o início, a pesquisa acadêmica e a formação de RHs, que evoluíram de forma positiva no país (PACHECO, 2007), negligenciando os interesses e a participação do setor empresarial. Raramente as empresas foram identificadas como alvo dos programas de C\&T, sendo reservado ao setor produtivo o papel de absorvedor das ofertas de conhecimentos e de RHs gerados pelas instituições de ensino e pesquisa (VIOTTI, 2008; ARBIX; CONSONI, 2011).

Na década de 1990, o atual Ministério da Ciência, Tecnologia e Inovação (MCTI) estruturou um conjunto de esquemas para promover o dispêndio privado em P\&D e a interação com a pesquisa pública, como o Programa de Apoio à Capacitação Tecnológica da Indústria (Pacti). O programa agregava instrumentos novos e preexistentes em torno da interação U-E. Entretanto, as açôes para estimular o investimento privado em $\mathrm{P} \& \mathrm{D}$, bem como aquelas para promover as interaçôes entre as empresas e o setor público, tiveram sucesso muito limitado, em vista da ausência de unidades próprias de P\&D no setor produtivo (VELHO; SAENZ, 2002).

Entre 1999 e 2002 foram criados os Fundos Setoriais com o objetivo do desenvolvimento científico e tecnológico de determinado setor. $\mathrm{O}$ acesso aos Fundos Setoriais pode envolver projetos cooperativos com universidades/IPPs, tanto na condição de executante como de interveniente, crédito em condições mais favoráveis e subvenções. Particularmente, o Fundo Verde-Amarelo (FVA), instituído em 2000 (Lei n. 10.168/00; MP 2.159-70) constitui uma das ações de estímulo à interação U-E no Brasil, criando uma fonte estável de recursos para programas cooperativos (PACHECO, 2007). Pereira (2005) analisou o impacto do FVA no período 1999-2003, destacando sua capacidade de mobilizar um grande número de empresas, bem como o uso de outros mecanismos de financiamento à empresa, como a Carta Convite. Ademais, o FVA contemplou setores com cobertura insatisfatória ou inexistente em outros Fundos Setoriais, como os setores petroquímico, siderúrgico e metal-mecânico.

Araujo et al. (2012), avaliando o impacto dos Fundos Setoriais no período 2001- 2006, verificaram um impacto positivo e significativo no pessoal ocupado total, mas marginal nas exportaçôes de alto conteúdo tecnológico. Além disso, os autores observaram que projetos cooperativos apresentavam impactos menos signi- 
ficativos (ou nulos) sobre o pessoal ocupado técnico-científico das empresas do que a concessão de crédito em condições mais favoráveis.

Analisando o impacto sobre a produtividade científica dos pesquisadores universitários envolvidos em projetos de pesquisa financiados pelos Fundos Setoriais, Kannebley et al. (2013) encontraram, em termos gerais, um efeito positivo, porém limitado, desta política. O efeito na produção acadêmica dos pesquisadores no período 2000-2008 esteve concentrado nas áreas de Engenharias e Ciências da Saúde e Exatas e da Terra e teve como meio principal de divulgação a publicação em periódicos nacionais.

O marco legal que possibilitou a concessão de subvenção econômica foi estabelecido a partir da aprovação da Lei da Inovação (Lei n. 10.973, de 02/12/2004) e da Lei do Bem (Lei n. 11.196, de 21/11/2005). Estas leis criaram instrumentos de apoio às empresas em várias dimensōes: multiplicação dos mecanismos de incentivos fiscais à $\mathrm{P} \& \mathrm{D}$ com aplicação automática; programas de subsídios para projetos de desenvolvimento tecnológico; mecanismos de subsídios para fixação de pesquisadores em empresas; e programas de financiamento para inovação com capital de risco (ARBIX; CONSONI, 2011, p. 215).

A aprovação da Lei de Inovação constituiu uma inflexão na trajetória do sistema de gestão da propriedade intelectual e de transferência de tecnologia na universidade brasileira, ao proporcionar amparo legal e definir incentivos para a comercialização dos resultados das pesquisas científicas e tecnológicas (ARBIX; CONSONI, 2011). Nesse novo marco encontra-se a obrigatoriedade de criação dos NITs, uma unidade responsável por gerir as políticas de inovação nas ICTs. Adicionalmente, a Lei de Inovação trouxe avanços importantes sobre o licenciamento de tecnologias, com diretrizes que orientam a forma como os royalties devem ser distribuídos na universidade.

Arbix e Consoni (2011) identificaram que, nos primeiros cinco anos após a aprovação da Lei de Inovação, dezenas de NITs foram criados no Brasil. Em investigação da trajetória de mudanças institucionais relacionadas com a construção de NITs na Universidade Estadual de Campinas (Unicamp), na Universidade de São Paulo (USP) e na Pontifícia Universidade Católica do Rio Grande do Sul (PUC-RS), os autores identificaram que todas as atividades referentes à proteção da propriedade intelectual e à transferência de know-how foram aceleradas. Adicionalmente, ocorreu um relevante aumento dos pedidos de patente depositados no Instituto Nacional de Propriedade Industrial (Inpi) após a criação das agências nas referidas universidades. 
Avaliando os impactos da Lei do Bem sobre as atividades inovativas das empresas usuárias deste incentivo fiscal, Calzolaio e Dathein (2012) constataram que se trata de um instrumento adequado para intensificar no curto prazo as atividades de inovação das empresas que já inovam, embora não seja apropriado para ampliar o número de empresas inovadoras e para apoiar projetos arriscados, que necessitam de grande aporte de capital e comprometem os custos de longo prazo. Para as empresas usuárias da Lei do Bem, os autores identificaram: aumento significativo do número daquelas que atribuíram alto grau de importância à cooperação com universidades e IPPs para a inovação; redução intensa do número de empresas que negligenciavam a importância da cooperação com universidades e institutos de pesquisa; e elevação dos dispêndios com $\mathrm{P} \& \mathrm{D}$ interno e a aquisição de $\mathrm{P} \& \mathrm{D}$ externo no conjunto das empresas usuárias do benefício fiscal.

Outra importante iniciativa em nível federal para o estímulo à cooperação U-E é o programa RHAE Pesquisador na Empresa. ${ }^{1}$ Desde 2007, este programa é destinado à inserção de pesquisadores (mestres e doutores) em empresas privadas, preferencialmente de micro, pequeno e médio portes. Além da possibilidade de aumentar a fixação de pesquisadores nas empresas, espera-se favorecer uma maior interação U-E por meio da relação pesquisador-empresário (CGEE, 2011). Teixeira e Menezes (2013), em avaliação de impacto do Programa RHAE Pesquisador na Empresa em três chamadas públicas, ${ }^{2}$ identificaram que ele é exitoso ao contribuir para a fixação de mestres e doutores nas empresas, promovendo também a capacitação de RHs em escala considerável e a geração de empregos de qualidade. Adicionalmente, o programa incentiva a inovação nas empresas ao induzir o desenvolvimento de projetos de P\&D\&I e a presença de mestres e doutores nas empresas favorece a relação U-E no tocante à transferência de tecnologia.

Iniciativas de fomento à cooperação U-E também vêm sendo desenvolvidas pelas fundações estaduais de amparo à pesquisa (FAPs). Algumas delas, por meio da Financiadora de Estudos e Projetos (Finep), implantaram, em 2003, o Programa de Apoio à Pesquisa em Empresas (Pappe), para apoiar financeiramente projetos inovadores que fossem desenvolvidos em conjunto por empresas e pesquisadores, contando com recursos da Finep e com contrapartida das FAPs e das empresas. Em 2006, o repasse de recursos passou a ser realizado diretamente às empresas, com a

1 O Programa de Formação de Recursos Humanos em Áreas Estratégicas (RHAE), criado em 1987 com gestão do MCTI e execução realizada pelo CNPq, utiliza um conjunto de modalidades de bolsas de fomento tecnológico, especialmente criado para agregar pessoal altamente qualificado em atividades de P\&D nas empresas, além de formar e capacitar RHs que atuem em projetos de pesquisa aplicada ou de desenvolvimento tecnológico.

2 Sendo: $32 / 2007,67 / 2008$ e 62/2009. 
denominação Pappe Subvenção, assumindo um direcionamento explícito às micro e pequenas empresas.

Carrijo e Botelho (2013), analisando o Programa Pappe, observaram que as relaçōes de cooperação já eram realizadas de forma significativa antes da participação no programa, principalmente com clientes/consumidores, universidades e IPPs. A relação de parceria com o pesquisador, exigência para a participação no programa, também já ocorria previamente à seleção pelo Pappe, sugerindo que o programa não tende a originar novas parcerias, mas sim a manter e/ou fortalecer as já existentes.

Em relação à abrangência e efetividade dos atuais programas de apoio à inovação direcionados às pequenas empresas brasileiras, Avellar e Botelho (2015) identificaram que aquelas que receberam incentivos financeiros apresentam, além de indicadores de esforço inovativo mais elevados, fontes mais diversificadas para inovação (maior participação em arranjos cooperativos, participação em incubadoras e realização de P\&D contínuo). A elevada participação de pequenas empresas beneficiadas pelos incentivos financeiros em arranjos cooperativos seria um indicativo da efetividade do suporte público na realização de parcerias e formação de redes de empresas. Adicionalmente, as autoras encontraram uma grande participação das empresas de pequeno porte em instrumentos de apoio à inovação, como participação em projetos com parceria e bolsas de apoio a pesquisadores.

Rapini et al. (2014) compararam empresas que financiaram com recursos próprios e com uma composição de recursos públicos e próprios os projetos em colaboração com universidades/IPPs, na busca de inferir especificidades ou não da interação. A análise dos dois conjuntos de empresas apontou que a natureza do financiamento da cooperação parece não interferir nas fontes de informação e nos modos de interação, mas sim nos objetivos (ou na motivação) da cooperação U-E. O financiamento exclusivo com recursos privados esteve mais voltado a resultados que contribuem diretamente na solução dos problemas da empresa e que são de maior apropriação (testes para produtos e processos; transferência de tecnologia). Já a composição de recursos públicos e privados está vinculada a projetos cooperativos de maiores risco e custo (projetos em conjunto).

\section{Base de dados e metodologia}

\subsection{Base de dados: Diretórios dos Grupos de Pesquisa do CNPq}

O DGP/CNPq reúne informações sobre os GPs em atividade no país, englobando pesquisadores, estudantes, técnicos, linhas de pesquisa em andamento e a produção 
científica, tecnológica e artística (CT\&A) gerada pelos grupos. Apesar de ser uma base de preenchimento opcional, seu universo abrangido vem aumentando ao longo dos anos, podendo-se supor relativa representatividade da comunidade científica nacional. As universidades, instituições de ensino superior e institutos que ministram cursos de pós-graduação concentram mais de $90 \%$ dos GPs cadastrados, não fazendo parte do DGP as empresas privadas.

Para este artigo, foram utilizados os microdados do DGP do Censo de 2008 oriundos do Projeto MCTI/Finep "Metodologia de Avaliação dos Resultados de Conjuntos de Projetos Apoiados por Fundos de Ciência, Tecnologia e Inovação (C,T\&I)" ${ }^{3}$ Tais dados permitem analisar, pela primeira vez, as interaçôes entre os modos de interação (ou tipos de relacionamento) e os tipos de remuneração. Isso justifica não terem sido empregadas as informações do Censo mais recente de 2010, cujo acesso no site apenas disponibiliza as informações agregadas.

Para o presente trabalho foram considerados os GPs cadastrados no DGP/CNPq, cujos líderes declararam algum relacionamento com o setor produtivo. Há dez tipos de remuneração entre os GPs e o setor produtivo que estão listados no Quadro 1.

\section{Quadro 1}

Tipos de remuneração entre os grupos de pesquisa e o setor produtivo

\begin{tabular}{|c|l|}
\hline 1 & Transferência de recursos financeiros do parceiro para o grupo \\
\hline 2 & Transferência de recursos financeiros do grupo para o parceiro \\
\hline 3 & $\begin{array}{l}\text { Parceria sem a transferência de recursos envolvendo exclusivamente relacionamento de } \\
\text { risco }\end{array}$ \\
\hline 4 & Fornecimento de bolsas para o grupo pelo parceiro \\
\hline 5 & Transferência de insumos materiais para as atividades de pesquisa do grupo \\
\hline 6 & Transferência de insumos materiais para as atividades do parceiro \\
\hline 7 & Transferência física temporária de $\mathrm{RH}$ do parceiro $\mathrm{p} /$ atividades de pesquisa do grupo \\
\hline 8 & Transferência física temporária de $\mathrm{RH}$ do grupo para as atividades do parceiro \\
\hline 9 & Parceria com transferência de recursos nos dois sentidos \\
\hline 10 & Outras formas de remuneração que não se enquadrem nas anteriores \\
\hline
\end{tabular}

Fonte: DGP/CNPq.

Cada líder do grupo pode atribuir até três tipos de remuneração decorrentes de relacionamentos interativos com o setor produtivo. Observa-se que a remuneração abrange não apenas recursos financeiros, mas também a transferência de conhecimento, RHs e insumos materiais. As parcerias podem ainda ser remuneradas por

3 Projeto desenvolvido no âmbito do convênio MCTI/Finep/UFMG/Ipea, que teve por objetivo desenvolver e aplicar metodologia qualitativa e quantitativa para avaliação do conjunto de projetos apoiados por fundos públicos de CT\&I no Brasil. 
meio de bolsas, ou não serem remuneradas, envolvendo exclusivamente relacionamentos de risco.

\subsection{Metodologia: modelo logit multinomial}

Para a análise de características relevantes dos GPs, das empresas e das interações que influenciam no tipo de remuneração estabelecida, foi proposto um modelo econométrico logit multinomial. ${ }^{4}$ Tal escolha metodológica recai justamente sobre a possibilidade de realizar análises comparativas entre os distintos tipos de remuneração.

A lógica de análise dos estimadores do modelo logit multinomial é a indicação da direção ou chance das probabilidades do grupo de análise $\mathrm{X}_{1 \mathrm{j}}$ em relação aos demais grupos de controle $\mathrm{X}_{\mathrm{k}}$, de forma que coeficientes estimados negativos expressam diminuição da probabilidade de se pertencer ao grupo de análise em relação ao grupo de controle, e estimadores positivos representam exatamente o contrário.

Empiricamente, portanto, o modelo logit multinomial permite aferir a probabilidade de uma interação ser remunerada em determinado tipo vis-à-vis outro tipo conforme características (variáveis) específicas dos grupos, interaçōes e empresas.

Ainda, como forma de melhor entendimento sobre os resultados, pode-se proceder com a análise da taxa relativa de risco (TRR), que é a probabilidade de escolha do grupo de análise sobre a probabilidade de escolha do grupo de controle, podendo ser obtida pela transformação exponencial dos estimadores lineares conseguidos pelo método de máxima verossimilhança.

A seguir, apresentam-se as variáveis consideradas para esse trabalho.

\section{Variável dependente: remuneração dos relacionamentos}

Como a técnica logit multinomial apresenta resultados relativos de comparação entre os diferentes grupos em relação ao grupo de controle, proceder com a análise de dez diferentes tipos de remuneração torna-se inviável. Assim, optou-se pela definição de três grupos: remuneração via recursos financeiros e materiais (grupo de controle), correspondente às remunerações 1, 2, 5, 6 e 9 do Quadro 1; remuneração via conhecimento, referente aos tipos 4, 7 e 8 do Quadro 1; e remuneração de risco, item 3 do Quadro 1).

As interações remuneradas como "outros tipos de remuneração" (tipo 10 do Quadro 1) foram excluídas da análise por representarem um grupo heterogêneo e

4 Para a apresentação técnica do modelo logit multinomial, ver Greene (2003). 
desconhecido de remunerações, não permitindo aferir nenhuma conclusão sobre seus resultados. Ademais foi considerada apenas a primeira remuneração informada pelo líder do GP.

\section{Variáveis independentes}

a) Excelência cientifica - fator obtido pelo método de análise fatorial $(\mathrm{AF})^{5}$ para variáveis relativas aos GPs que interagiram, todas disponibilizadas na base do DGP. As variáveis utilizadas para a definição do indicador ${ }^{6}$ são: idade do GP; número de doutores atuantes no GP; quantidade de artigos de circulação nacional publicados; quantidade de artigos de circulação internacional publicados; número de teses defendidas por membros do grupo; e número de dissertaçôes defendidas por membros do grupo.

Alguns trabalhos indicam que a interação U-E é fortemente influenciada pela quantidade e qualidade da pesquisa científica e que as empresas inovadoras utilizam com mais frequência a pesquisa universitária de alta qualidade e em periódicos de qualidade (MANSFIELD; LEE, 1996; NARIN et al., 1997).

b) Grande área - variável dummy para as oito grandes áreas do conhecimento classificadas no DGP. Para a análise, será utilizada a grande área Ciências Agrárias como unidade de comparação.

Alguns trabalhos também indicam a especificidade da área ou do setor na interação U-E (KLEVORICK et al., 1995; MEYER-KRAMER; SCHMOCH, 1998; COHEN et al., 2002), bem como o tipo de interação estabelecida (SCHARTINGER et al., 2002).

c) Tipo de relacionamento - os relacionamentos da base foram reclassificados em duas formas diferentes: relacionamento bidirecional, que ocorre em pesquisa científica de uso imediato e sem uso imediato dos resultados; e relacionamento unidirecional, referente à transferência de tecnologia, desenvolvimento de software, engenharia não rotineira, consultoria e treinamento (ver Tabela 2).

O intuito dessa classificação é a diferenciação dos relacionamentos que envolvem trocas bidirecionais de informação e conhecimento entre os agentes daqueles que apresentam apenas uma direção unívoca de prestação de serviços ou de desenvolvi-

5 Para mais informaçōes sobre o método de análise fatorial, ver Mingoti (2005).

6 A proporção acumulada de explicação do fator 1 obtido pelo método de AF para o conjunto de variáveis sugeridas foi de 101,46\%, o que corrobora sua validade para o objetivo proposto de expressar a relevância científica do GP. 
mento de tecnologias e produtos. Nesse caso, o relacionamento de controle é o bidirecional, sendo excluídos da análise os grupos que indicaram "outros tipos de relacionamento" na base CNPq. Assim como no caso dos tipos de remuneração, o líder do GP pode atribuir até três tipos de relacionamento, mas foi considerada apenas a primeira opção informada.

Esta escolha é corroborada por alguns trabalhos que verificaram que distintos modos de interação podem estar associados a diferentes fontes de financiamento. Jensen et al. (2010) observaram que a consultoria está positivamente associada ao financiamento do governo. Já Muscio et al. (2013) encontraram complementaridade de financiamento público e privado nos contratos de pesquisa e nas consultorias.

d) Empresas - variável dummy de controle dos tipos de empresas que participaram da interação com os GPs, com as seguintes tipologias setoriais: setores baseados na ciência; empresas dominadas por fornecedores; fornecedores especializados; intensivos em escala; comércio e serviços; administração pública; e intermediação financeira.

A classificação é feita pelo nível de intensidade tecnológica setorial, de acordo com Campos e Urraca Ruiz (2009), para os quatro primeiros tipos de empresas classificadas. Os três últimos tipos foram classificaçôes definidas pelos autores do artigo, no sentido de compatibilizar as informações disponibilizadas na base do DGP. Para essa classificação, a dummy de controle é estabelecida para as empresas pertencentes aos setores baseados em ciência.

\section{Modos de interação e de remuneração dos grupos de pesquisa com o setor produtivo}

Do total dos GPs cadastrados no Diretório em 2008, 2.726, cerca de 12\% do total, relataram algum tipo de relacionamento com o setor produtivo. Estes grupos são filiados a 272 instituições. A Tabela 1 traz o total de GPs e os grupos interativos, segundo grandes áreas do conhecimento, bem como o total de relacionamentos e da remuneração. Os grupos interativos foram responsáveis por 10.423 relacionamentos com o setor produtivo, os quais tiveram 8.626 remuneraçóes, indicando que diferentes tipos de relacionamento podem ter sido remunerados de uma mesma forma. As Tabelas 2 e 3 apresentam, respectivamente, os diferentes tipos de relacionamento e de remuneração. 
Tabela 1

Grupos de pesquisa, total e com relacionamento com o setor produtivo, e total de relacionamentos, segundo grandes áreas do conhecimento Brasil - 2008

\begin{tabular}{l|c|c|c|c|c}
\hline $\begin{array}{c}\text { Grandes áreas do } \\
\text { conhecimento }\end{array}$ & $\begin{array}{c}\text { Grupos de } \\
\text { pesquisa } \\
\text { total (1) }\end{array}$ & $\begin{array}{c}\text { Grupos com } \\
\text { relaciona- } \\
\text { mento } \mathbf{( 2 )}\end{array}$ & $\begin{array}{c}(\mathbf{2}) /(\mathbf{1}) \\
\mathbf{( \% )}\end{array}$ & $\begin{array}{c}\text { Total de } \\
\text { relaciona- } \\
\text { mentos }\end{array}$ & $\begin{array}{c}\text { Total } \\
\text { de remu- } \\
\text { neraçáo }\end{array}$ \\
\hline Ciências Agrárias & 2.699 & 521 & 19,3 & 2.228 & 1.791 \\
Ciências Biológicas & 2.696 & 276 & 10,2 & 795 & 715 \\
Ciências da Saúde & 3.961 & 332 & 8,4 & 938 & 828 \\
Ciências Exatas e da Terra & 2.515 & 429 & 17,1 & 527 & 798 \\
Engenharias & 3.027 & 880 & 29,1 & 4.343 & 3.482 \\
Ciências Humanas & 5.387 & 181 & 3,35 & 527 & 426 \\
Ciências Sociais Aplicadas & 3.438 & 220 & 6,4 & 591 & 529 \\
Linguística, Letras e Artes & 1.836 & 30 & 1,6 & 62 & 57 \\
Total & $\mathbf{2 2 . 7 9 7}$ & $\mathbf{2 . 7 2 6}$ & $\mathbf{1 1 , 9}$ & $\mathbf{1 0 . 4 2 3}$ & $\mathbf{8 . 6 2 6}$ \\
\hline
\end{tabular}

Fonte: DGP/CNPq, Censo 2008.

No geral, as áreas de Ciências Humanas e Ciências da Saúde reúnem maior número de GPs cadastrados no DGP/CNPq, com, respectivamente, 5.387 (23,6\% do total) e $3.961(17,4 \%)$. Por sua vez, as áreas que apresentaram maior número de grupos interativos foram as de Engenharias (29,1\%), Ciências Agrárias (19,3\%) e Ciências Exatas e da Terra (17,1\%). Estas também foram as áreas que registraram maior número de relacionamentos, sendo que a de Engenharias reuniu mais de $1 / 3$ do total ( 4.081 ou $35 \%$ do total). A remuneração segue o padrão identificado anteriormente, concentrando-se nas áreas de Engenharias (40,3\% do total) e Ciências Agrárias (20,7\%), que em conjunto responderam por cerca de $60 \%$ do total dos tipos de remuneração entre os GPs e o setor produtivo. Este cenário remonta a especificidades brasileiras e setoriais. A área de Ciências Agrárias vem sendo apoiada e fomentada por políticas do governo, em função da orientação exportadora do agronegócio do país, contando com a importante presença da Embrapa. Já as Engenharias são tradicionalmente áreas cujos resultados são próximos da realidade industrial.

Segundo os dados da Tabela 2, os modos de interação mais frequentes, que partem dos GPs para o setor produtivo, são pesquisa científica com uso imediato dos resultados (podendo-se considerar como pesquisa de curto prazo), com cerca de $30 \%$ do total dos relacionamentos, transferência de tecnologia $(16,4 \%)$ e pesquisa científica sem uso imediato dos resultados (podendo-se considerar pesquisa de longo prazo) $(14,3 \%)$. Estes três tipos de relacionamento abarcam 60\% do total. Por sua 
vez, entre os relacionamentos que partem do setor produtivo para os GPs, ainda que em menor magnitude, os mais frequentes foram transferência de tecnologia $(2,8 \%)$ e treinamento de pessoal (1,9\%). Estes dados indicam que as colaborações entre os GPs e o setor produtivo, conforme indicado pelos líderes, envolvem não apenas um fluxo unidirecional, mas também bidirecional de informação e conhecimento, justificando, pois, a separação dos mesmos no modelo.

Tabela 2

Modos de interação entre GPs e o setor produtivo

Brasil - 2008

\begin{tabular}{l|r|r}
\hline \multicolumn{1}{c}{ Modos de interação de acordo com a origem } & \multicolumn{2}{c}{ Relacionamentos } \\
\cline { 2 - 3 } & $\mathbf{N}$ & $\mathbf{\%}$ \\
\hline Grupos de pesquisa-> Setor produtivo & 776 & 7,4 \\
Consultoria técnica & 334 & 3,2 \\
Engenharia não rotineira (1) & 281 & 2,7 \\
Desenvolvimento de software & 3.061 & 29,4 \\
Pesquisa científica com uso imediato & 1.488 & 14,3 \\
Pesquisa científica sem uso imediato & 1.710 & 16,4 \\
Transferência de tecnologia & 612 & 5,9 \\
Treinamento de pessoal & 665 & 6,4 \\
Insumos materiais & 698 & 6,7 \\
Outros & & 0,0 \\
Setor produtivo-> Grupos de pesquisa & 130 & 1,2 \\
Engenharia não rotineira (2) & 119 & 1,1 \\
Desenvolvimento de software & 289 & 2,8 \\
Transferência de tecnologia & 196 & 1,9 \\
Treinamento de pessoal & 64 & 0,6 \\
Insumos materiais & $\mathbf{1 0 . 4 2 3}$ & $\mathbf{1 0 0 , 0}$ \\
Total de relacionamentos & & \\
\hline
\end{tabular}

Fonte: DGP/CNPq, Censo 2008.

(1) Inclusive o desenvolvimento de protótipo cabeça de série ou planta-piloto para o parceiro.

(2) Inclusive o desenvolvimento de fabricação de equipamentos para o grupo.

A Tabela 3 apresenta os tipos de remuneração da interação dos GPs com o setor produtivo. As remunerações foram agrupadas em quatro blocos de acordo com características afins. Um primeiro bloco reúne recursos para a pesquisa, que podem ser financeiros ou insumos materiais. O segundo agrupa remunerações associadas à geração e transmissão de conhecimento entre as partes, seja por meio de bolsas ou da transferência física e temporária de recursos humanos entre os GPs e o setor 
produtivo. O terceiro é o único relacionamento que envolve apenas risco e o quarto abrange os outros tipos de remuneração. ${ }^{7}$ A transferência de recursos financeiros e materiais foi o tipo de remuneração mais frequente, respondendo por $56,7 \%$ do total dos tipos de relacionamentos. Nesse grupo, destacaram-se a transferência de recursos financeiros do parceiro para o grupo, reunindo $30,2 \%$ do total, e a transferência de insumos materiais para as atividades de pesquisa do grupo (18,1\%). Menos frequentes foram as transferências de recursos financeiros e de insumos materiais do grupo para o setor produtivo, com, respectivamente $2,1 \%$ e $1,8 \%$.

Tabela 3

Tipos de remuneração da interação dos grupos de pesquisa com o setor produtivo

Brasil - 2008

\begin{tabular}{|c|c|c|c|}
\hline \multirow{2}{*}{ Tipos } & \multirow{2}{*}{ Remuneração } & \multicolumn{2}{|c|}{ Relacionamentos } \\
\hline & & $\mathbf{N}$ & $\%$ \\
\hline \multirow{6}{*}{$\begin{array}{l}\text { Recursos } \\
\text { financeiros e } \\
\text { materiais }\end{array}$} & $\begin{array}{l}\text { Transferência de recursos financeiros do parceiro para o } \\
\text { grupo }\end{array}$ & 2.606 & 30,2 \\
\hline & $\begin{array}{l}\text { Transferência de insumos materiais para as atividades de } \\
\text { pesquisa do grupo }\end{array}$ & 1.562 & 18,1 \\
\hline & Parceria com transferência de recursos nos dois sentidos & 379 & 4,4 \\
\hline & $\begin{array}{l}\text { Transferência de recursos financeiros do grupo para o } \\
\text { parceiro }\end{array}$ & 184 & 2,1 \\
\hline & $\begin{array}{l}\text { Transferência de insumos materiais para as atividades do } \\
\text { parceiro }\end{array}$ & 159 & 1,8 \\
\hline & Total & 4.890 & 56,7 \\
\hline \multirow{4}{*}{$\begin{array}{l}\text { Troca de } \\
\text { conhecimento }\end{array}$} & Fornecimento de bolsas para o grupo pelo parceiro & 1.080 & 12,5 \\
\hline & $\begin{array}{l}\text { Transferência física temporária de } \mathrm{RH} \text { do parceiro para as } \\
\text { atividades de pesquisa do grupo }\end{array}$ & 379 & 4,4 \\
\hline & $\begin{array}{l}\text { Transferência física temporária de } \mathrm{RH} \text { do grupo para as } \\
\text { atividades do parceiro }\end{array}$ & 226 & 2,6 \\
\hline & Total & 1.685 & 19,5 \\
\hline Risco & $\begin{array}{l}\text { Parceria sem a transferência de recursos envolvendo } \\
\text { exclusivamente relacionamento de risco }\end{array}$ & 1.126 & 13,1 \\
\hline \multirow[t]{2}{*}{ Outras } & $\begin{array}{l}\text { Outras formas de remuneração que não se enquadrem nas } \\
\text { anteriores }\end{array}$ & 925 & 10,7 \\
\hline & Total geral & 8.626 & 100,0 \\
\hline
\end{tabular}

Fonte: DGP/CNPq, Censo 2008.

É relevante destacar a importância das remunerações relacionadas à geração e troca de conhecimento, que responderam, em conjunto, por 19,5\% do total. As

7 Um teste com a aplicação de análise de correspondência múltipla permitiu inferir que pesquisas de longo prazo, no geral, são remuneradas com parcerias de risco, bolsas e transferência de pessoal. Ademais, recursos financeiros do setor produtivo foram utilizados para remunerar principalmente pesquisas científicas de curto prazo, tecnologia e software desenvolvidos pelo grupo e atividades de consultoria. 
atividades de pesquisa geram conhecimento muitas vezes de natureza tácita, não transmissível, o que pode explicar a relevância da transferência temporária de pessoal entre as partes. A transferência temporária de pessoal do parceiro para o grupo $(4,4 \%)$ foi mais frequente do que a do grupo para o parceiro $(2,6 \%)$. Enquanto o primeiro tipo de remuneração pode abranger funcionários das empresas fazendo cursos, mestrado ou doutorado nas universidades, o segundo pode incluir estágio de estudantes ou mesmo a permanência de pesquisadores nas empresas, durante período de vigência do projeto/pesquisa.

Em terceiro lugar estão as parcerias que envolvem apenas risco sem a transferência de recursos de qualquer espécie $(13,0 \%)$ e, em quarto, o fornecimento de bolsas para o grupo $(12,5 \%)$.

\section{Resultados do modelo logit multinomial}

No modelo logit multinomial os coeficientes são uma comparação da probabilidade de se estar em um grupo de análise em relação a um grupo de controle (no caso, a remuneração via recursos financeiros e materiais). A análise das Tabelas 4 e 5 segue essa forma de verificação. Cabe destacar que os resultados dessas tabelas fazem parte do mesmo modelo logit multinomial e só foram separados para facilitar a análise comparativa. Na Tabela 4, a comparação é realizada entre o grupo de controle (remuneração recursos financeiros e materiais) e a remuneração conhecimento. $\mathrm{Na}$ Tabela 5, é feita a análise do grupo de controle em relação à remuneração de risco.

Para o modelo foram considerados apenas o primeiro relacionamento e a primeira remuneração atribuídos pelos líderes dos grupos de pesquisa, supondo-se serem os mais relevantes. Não há uma equivalência direta entre os tipos de relacionamento e de remuneração, de forma que qualquer outro procedimento iria requer elevado grau de arbitrariedade.

A base de dados analisada no modelo é composta de 6.557 interações. Conforme mencionado na seção 3.2, foram excluídas as interações que apresentaram as classificaçôes "outros tipos de relacionamento" (373 observações) e "outros tipos de remuneração" (719 observaçôes). Ademais, em 895 interações não foi possível identificar o setor da empresa, sendo as mesmas também excluídas da análise. Cabe assinalar que 428 interações são intercessão das três categorias excluídas. Após estes procedimentos, a base de informações para o modelo logit multinomial foi composta de 4.998 interações realizadas entre os GPs e o setor produtivo. 
Tabela 4

Resultados do modelo logit multinomial - Comparação 1

Variável dependente: comparativo escolha remuneração conhecimento em relação a remuneração recursos financeiros e materiais

Interação comparativa (dummies):

Grande área: Ciências Agrárias; relacionamento: canal bidirecional; empresas: baseadas em ciência

\begin{tabular}{|c|c|c|c|}
\hline \multicolumn{4}{|l|}{ Número de observações: 4.998} \\
\hline & Coeficiente & p-valor & TRR \\
\hline Excelência científica & $-0,2628$ & 0,000 & 0,7688 \\
\hline \multicolumn{4}{|l|}{ Grandes áreas } \\
\hline Ciências Biológicas & 0,1521 & 0,306 & 1,1642 \\
\hline Ciências da Saúde & 0,2805 & 0,045 & 1,3238 \\
\hline Ciências Exatas e da Terra & 0,0489 & 0,715 & 1,0501 \\
\hline Ciências Humanas & 0,7381 & 0,000 & 2,0920 \\
\hline Ciências Sociais Aplicadas & 0,7260 & 0,000 & 2,0667 \\
\hline Engenharias & $-0,5921$ & 0,000 & 0,5532 \\
\hline Linguística, Letras e Artes & 0,6028 & 0,149 & 1,8273 \\
\hline \multicolumn{4}{|l|}{ Tipo de relacionamento } \\
\hline Unidirecional & $-0,0793$ & 0,058 & 0,9237 \\
\hline \multicolumn{4}{|l|}{ Empresas } \\
\hline Dominadas por fornecedores & $-0,2771$ & 0,043 & 0,7580 \\
\hline Fornecedores especializados & $-0,5239$ & 0,002 & 0,5922 \\
\hline Intensivas em escala & $-1,0786$ & 0,000 & 0,3401 \\
\hline Comércio e prestação de serviços & 0,5274 & 0,000 & 1,6947 \\
\hline Administração pública & 0,7022 & 0,000 & 2,0183 \\
\hline Intermediação financeira & $-0,4638$ & 0,285 & 0,6289 \\
\hline Constante & $-1,3940$ & 0,000 & 0,2480 \\
\hline \multicolumn{4}{|l|}{ LR (qui-quadrado) $=660,62$} \\
\hline Prob $>$ qui-quadrado $=0.0000$ & & & \\
\hline
\end{tabular}

Fonte: DGP/CNPq, Censo 2008. Elaboração própria.

O valor da estatística qui-quadrado comprova a significância do modelo proposto. $\mathrm{O}$ primeiro resultado é concernente à relevância da excelência científica dos GPs. O modelo indica que um incremento no fator científico reduz a proba- 
bilidade relativa de se estabelecer remuneração via conhecimento vis-à-vis recursos financeiros e materiais; ou seja, grupos que possuem maior relevância científica têm probabilidade maior de serem financiados por recursos financeiros.

Em relação às grandes áreas científicas, a probabilidade relativa de se financiar por conhecimento tácito versus recursos financeiros e materiais aumenta quando o grupo que interage pertence às grandes áreas de Ciências da Saúde, Ciências Humanas e Ciências Sociais Aplicadas vis-à-vis Ciências Agrárias, e diminui quando o grupo que interage pertence à Engenharia vis-à-vis Ciências Agrárias. Os grupos de pesquisa das grandes áreas Ciências Biológicas, Ciências Exatas e da Terra e Linguística, Letras e Artes não apresentam coeficientes com significância estatística para diferenciá-los em relação aos grupos das Ciências Agrárias.

Há significância estatística na comparação dos resultados para os relacionamentos unidirecional e bidirecional. A probabilidade relativa de se financiar por conhecimento versus recursos financeiros e materiais diminui quando o relacionamento muda de bidirecional para unidirecional, ou seja, há uma probabilidade maior de se financiar por meio de conhecimento quando o relacionamento é bidirecional, isto é, quando envolve pesquisas de curto ou longo prazo.

$\mathrm{Na}$ classificação industrial, a probabilidade relativa de se financiar por conhecimento versus recursos financeiros e materiais diminui quando a empresa que interage é dos setores dominados por fornecedores, intensivos em escala e fornecedores especializados vis-à-vis empresas de setores baseados na ciência, e aumenta quando a empresa pertence aos setores de comércio e prestação de serviços e administração pública vis-à-vis empresas de setores baseados na ciência.

$\mathrm{Na}$ comparação entre o grupo de controle (remuneração via recursos financeiros e materiais) e remuneração via relacionamento de risco (Tabela 5), pode-se inferir que a excelência científica do GP diminui a probabilidade relativa de se estabelecer remuneração de risco vis-à-vis recursos financeiros e materiais. Portanto, na comparação direta dos resultados das Tabelas 4 e 5, é verificado que o incremento da excelência científica dos GPs aumenta a probabilidade de a remuneração da interação U-E ser estabelecida via recursos financeiros em comparação a todos os outros tipos de remuneração. Esse resultado evidencia a relevância da qualidade científica sobre as possibilidades de interação estabelecidas pelos GPs. A busca por parte dos grupos pela excelência acadêmica, expressa no incremento quantitativo e qualitativo de pesquisas, publicações e formação de $\mathrm{RH}$, aumenta as probabilidades de financiamentos por fontes de remuneração pecuniária direta. 
Tabela 5

Resultados do modelo logit multinomial - Comparação 2

Variável dependente: comparativo escolha remuneração risco em relação a remuneração recursos financeiros e materiais

Interação comparativa (dummies):

Grande área: Ciências Agrárias; relacionamento: canal bidirecional; empresas: baseadas em ciência

\begin{tabular}{|c|c|c|c|}
\hline \multicolumn{4}{|l|}{ Número de observações: 4.998} \\
\hline & Coeficiente & p-valor & TRR \\
\hline Excelência científica & $-0,3678$ & 0,000 & 0,6923 \\
\hline \multicolumn{4}{|l|}{ Grandes áreas } \\
\hline Ciências Biológicas & $-0,2340$ & 0,321 & 0,7914 \\
\hline Ciências da Saúde & $-0,0246$ & 0,909 & 0,9757 \\
\hline Ciências Exatas e da Terra & 0,2021 & 0,266 & 1,2239 \\
\hline Ciências Humanas & 1,7545 & 0,000 & 5,7807 \\
\hline Ciências Sociais Aplicadas & 0,5391 & 0,026 & 1,7144 \\
\hline Engenharias & 0,0708 & 0,636 & 1,0733 \\
\hline Linguística, Letras e Artes & $-14,9829$ & 0,991 & · \\
\hline \multicolumn{4}{|l|}{ Tipo de relacionamento } \\
\hline Unidirecional & $-0,6677$ & 0,000 & 0,5129 \\
\hline \multicolumn{4}{|l|}{ Empresas } \\
\hline Dominadas por fornecedores & $-0,5193$ & 0,003 & 0,5949 \\
\hline Fornecedores especializados & $-0,6610$ & 0,001 & 0,5163 \\
\hline Intensivas em escala & $-0,4561$ & 0,045 & 0,6338 \\
\hline Comércio e prestação de serviços & $-0,0689$ & 0,624 & 0,9333 \\
\hline Administração pública & 0,3623 & 0,028 & 1,4367 \\
\hline Intermediação financeira & $-15,3800$ & 0,984 & . \\
\hline Constante & $-1,8464$ & 0,000 & 0,1578 \\
\hline \multicolumn{4}{|l|}{ LR (qui-quadrado) $=660,62$} \\
\hline Prob $>$ qui-quadrado $=0.0000$ & & & \\
\hline
\end{tabular}

Fonte: DGP/CNPq, Censo 2008. Elaboração própria.

$\mathrm{Na}$ análise das grandes áreas do conhecimento, observa-se que a probabilidade relativa de se financiar por remuneração de risco versus recursos financeiros e materiais aumenta quando o grupo que interage pertence às áreas de Ciências Humanas e Sociais Aplicadas vis-à-vis Ciências Agrárias. Para todas as demais, os coeficientes 
não foram estatisticamente significantes, não sendo possível, portanto, diferenciá-las em relação às Ciências Agrárias.

Quanto aos tipos de relacionamento, a probabilidade relativa de se financiar por risco versus recursos financeiros e materiais diminui quando o relacionamento muda de bidirecional para unidirecional (ou seja, há uma probabilidade maior de se financiar por risco quando o relacionamento é bidirecional).

Analisando esse resultado em conjunto com os da Tabela 4, pode-se inferir que relacionamentos bidirecionais possuem maior probabilidade de serem financiados por conhecimento e risco vis-à-vis relacionamentos unidirecionais. Isso corrobora o que foi apresentado na seção 2 , no sentido de que projetos cooperados de pesquisas (relacionamento bidirecional) são financiados com expressiva participação do poder público.

O baixo investimento em atividades inovativas e a considerável aversão ao risco das empresas no Brasil remetem ao foco em interações, principalmente para resolução de problemas pontuais de adequação de processos e produtos, atribuindo baixa importância para relacionamentos duradouros com instituições de pesquisa que possam fornecer soluções inovadoras de maior valor agregado futuro.

$\mathrm{Na}$ comparação entre setores industriais, a probabilidade relativa de se financiar por risco versus recursos financeiros e materiais diminui quando a empresa que interage é dos setores dominados por fornecedores, intensivos em escala e fornecedores especializados vis-à-vis empresas de indústrias baseadas em ciência, e aumenta quando a empresa pertence à administração pública vis-à-vis empresas dos setores baseados na ciência.

Para confirmar os resultados do modelo, apresenta-se, na Tabela 6, a predição de probabilidades de escolha de cada tipo de remuneração para cada uma das variáveis, mantendo todas as outras variáveis do modelo no seu valor médio. Esta análise pode ser realizada apenas para variáveis explicativas categóricas (dummy).

A análise dos resultados deve ser feita da seguinte forma, por exemplo: se todas as variáveis estiverem em seu valor médio, um GP da grande área de Ciências Agrárias vai realizar em $74 \%$ das vezes relacionamentos com remuneração via recursos financeiros e materiais, $19 \%$ das vezes relacionamentos com remuneração do tipo conhecimento e $7 \%$ via remuneração de risco.

Dessa forma, para todas as variáveis, as maiores probabilidades estão associadas à remuneração via recursos financeiros e materiais. Tal resultado se expressa dessa forma porque $52,4 \%$ das remuneraçóes estabelecidas na base selecionada para a pesquisa são desse tipo. 
Tabela 6

Predição de probabilidades das variáveis categóricas, por tipo de remuneração

\begin{tabular}{|c|c|c|c|c|c|}
\hline Variáveis & $\begin{array}{c}\text { Recursos } \\
\text { financeiros } \\
\text { e materiais } \\
(\%)\end{array}$ & $\begin{array}{l}\text { Conheci- } \\
\text { mento }(\%)\end{array}$ & Risco (\%) & CT/RFM & R/RFM \\
\hline \multicolumn{6}{|l|}{ Grandes áreas } \\
\hline Ciências Agrárias & $0,740^{* *}$ & $0,190^{* *}$ & 0,070 & 0,257 & 0,095 \\
\hline Ciências Biológicas & $0,727^{*}$ & $0,218^{*}$ & 0,055 & 0,300 & 0,076 \\
\hline Ciências da Saúde & $0,698^{* *}$ & $0,237^{* *}$ & 0,065 & 0,340 & 0,093 \\
\hline Ciências Exatas e da Terra & $0,721^{* * *}$ & $0,195^{* * *}$ & 0,084 & 0,270 & 0,117 \\
\hline Ciências Humanas & $0,479^{* * *}$ & $0,258^{* * *}$ & 0,263 & 0,538 & 0,549 \\
\hline Ciências Sociais Aplicadas & $0,591^{* * *}$ & $0,314^{* * *}$ & 0,095 & 0,531 & 0,161 \\
\hline Engenharias & $0,804^{* * *}$ & $0,114^{* * *}$ & 0,082 & 0,142 & 0,102 \\
\hline Linguística, Letras e Artes & 0,681 & 0,319 & 0,001 & 0,469 & 0,001 \\
\hline \multicolumn{6}{|l|}{ Tipos de Relacionamento } \\
\hline Relacionamento bidirecional & 0,734 & 0,175 & 0,091 & 0,238 & 0,124 \\
\hline $\begin{array}{l}\text { Relacionamento } \\
\text { unidirecional }\end{array}$ & $0,779^{* *}$ & $0,172^{* *}$ & 0,050 & 0,221 & 0,064 \\
\hline \multicolumn{6}{|l|}{ Empresas } \\
\hline Baseados na ciência & $0,736^{* * *}$ & $0,161^{* * *}$ & 0,103 & 0,219 & 0,140 \\
\hline Dominadas por fornecedores & $0,801^{* *}$ & $0,133^{* *}$ & 0,066 & 0,166 & 0,082 \\
\hline Fornecedores especializados & $0,832^{*}$ & $0,107^{*}$ & 0,060 & 0,129 & 0,072 \\
\hline Intensivas em escala & $0,86^{* *}$ & $0,064^{* * *}$ & 0,076 & 0,074 & 0,088 \\
\hline $\begin{array}{l}\text { Comércio e prestação de } \\
\text { serviços }\end{array}$ & $0,666^{* * *}$ & $0,247^{* * *}$ & 0,087 & 0,371 & 0,131 \\
\hline Administração pública & 0,609 & 0,267 & 0,122 & 0,438 & 0,200 \\
\hline Intermediação financeira & $0,879^{*}$ & $0,121^{*}$ & 0,000 & 0,138 & 0,000 \\
\hline
\end{tabular}

Fonte: DGP/CNPq, Censo 2008. Elaboração própria.

Nota: Significância a $1 \%\left({ }^{*}\right), 5 \%\left({ }^{* *}\right)$ e $10 \%\left({ }^{* * *}\right)$, respectivamente.

$\mathrm{CT} / \mathrm{RFM}=$ Conhecimento/Recursos Financeiros e Materiais.

R/RFM = Risco/Recursos Financeiros e Materiais.

Porém, pela análise relativa das probabilidades (CT/RFM e R/RFM), mesmo que a probabilidade de se situar em remuneração via recursos financeiros e materiais seja maior para todas as características, os GPs das grandes áreas de Ciências Humanas e Ciências Sociais Aplicadas possuem maiores probabilidades de serem financiados via conhecimento e relacionamentos de risco $^{8}$ do que os grupos das

8 Essa constatação não vale para a área de Linguística, Letras e Artes para a remuneração "parcerias que enviolvem risco". 
demais grandes áreas. Esse resultado é congruente com o padrão de interação realizado por GPs dessas áreas, normalmente voltado para pesquisas científicas que não envolvem resultados de produtos/processos que originem retorno financeiro para os agentes.

Esse raciocínio também vale para empresas de comércio e prestação de serviços que, como se pode depreender na Tabela 6, apresentam índices maiores de remuneração por conhecimento do que os demais tipos de empresas.

Para empresas de setores baseados na ciência, pode-se notar também maior relevância para remuneração via conhecimento. Nesse caso, o resultado pode estar conectado: ao risco inerente ao processo de inovação setorial, direcionado para um maior grau de inovaçōes disruptivas, mas com menor probabilidade de sucesso; e a uma maior participação do setor público no financiamento dessas interações, justamente porque o risco inovativo setorial inibe a participação privada em projetos conjuntos de P\&D.

\section{Considerações finais}

Este artigo apresentou uma análise em caráter exploratório sobre a remuneração da interação U-E no Brasil, declarada pelos líderes dos GPs do CNPq. Os dados demonstraram que o tipo de remuneração mais frequente foi a transferência de recursos financeiros e materiais entre as partes, que respondeu por mais da metade $(52,4 \%)$ do total. Também relevantes são as remuneraçôes vinculadas à geração e troca de conhecimento, como bolsas e transferência de pessoal. Nesse sentido, os dados empíricos do DGP/CNPq confirmam as motivações para o engajamento em atividades cooperativas com universidades relacionadas ao acesso a recursos complementares, compartilhando conhecimentos e habilidades, bem como o risco das atividades de pesquisa.

As estimaçôes do modelo logit multinomial apontam que há diferenças estatísticas nas formas de remuneração estabelecidas entre os GPs e o setor produtivo no que tange à excelência científica dos grupos, às áreas do conhecimento, ao tipo de relacionamento estabelecido e à classificação setorial das empresas segundo o nível de intensidade tecnológica. Cabe ressaltar que, por características da base de dados, o modelo analisa um conjunto restrito de interações, a saber, a primeira resposta sobre o tipo de remuneração e o tipo relacionamento fornecida pelo líder do GP. Entende-se, porém, que essa limitação não se apresenta como uma restrição que inviabiliza a análise, sendo na verdade mais importante a subestimação dos resul- 
tados por essa via do que a inobservância da possibilidade de existência de uma classificação hierárquica sobre a ordem de respostas fornecidas pelos respondentes.

Pode-se constatar que o aumento da excelência científica dos GPs permite que se aumente a probabilidade de remuneração por meio de recursos financeiros e materiais vis-à-vis os demais tipos de remuneração. Esse resultado está relacionado diretamente à diminuição da incerteza inerente ao aumento da capacitação científica dos GPs. Quando se interage com grupos que possuem melhores resultados científicos, a possibilidade de se alcançarem os resultados esperados na interação torna-se maior, o que garante a possibilidade de remuneração pecuniária com maior probabilidade (GOLDFARB, 2008).

Em relação à comparação das grandes áreas, GPs das áreas de Humanidades (Ciências Humanas e Ciências Sociais Aplicadas), menos propensas a gerar inovações tecnológicas, tendem a estabelecer relacionamentos interativos por meio de remuneração baseada na geração e troca de conhecimento - intercâmbio de recursos humanos e bolsas de pesquisa - e via parcerias de risco com maiores probabilidades do que as demais grandes áreas. A área Ciências da Saúde também apresentou como relevante a remuneração via conhecimento. Por sua vez, a grande área de Engenharias registrou a maior probabilidade de estabelecimento de remuneração via recursos financeiros e materiais.

Do ponto de vista dos modos de interação, tem-se que as pesquisas científicas de curto e longo prazos (relacionamentos bidirecionais) apresentam maior probabilidade de serem financiadas por meio de parcerias que envolvem somente risco, de bolsas e da transferência de pessoal do grupo para o parceiro, envolvendo um menor comprometimento financeiro, do que as interações unidirecionais. Esse resultado aponta, portanto, a relevância do apoio do governo (por meio de bolsas de pesquisa e do programa de fixação e de capacitação de recursos humanos, como é caso do Programa RHAE) para a realização de pesquisas científicas de curto e longo prazos, que envolvem maior risco e incerteza quanto aos resultados esperados. Essa constatação é ainda confirmada pela análise das empresas classificadas de acordo com a intensidade tecnológica. Entre os resultados destaca-se a maior probabilidade de remuneração por conhecimento e risco para empresas de setores baseados na ciência do que os demais setores industriais da classificação de Campos e Urraca Ruiz (2009), bem como de setores de comércio e de prestação de serviços. Os setores baseados na ciência são aqueles cujas oportunidades tecnológicas, em grande parte, são oriundas de avanços no conhecimento científico, estando mais sujeitos às 
incertezas do processo de pesquisa. Já os setores de comércio e prestação de serviços possuem uma dinâmica inovativa distinta, decorrente de suas especificidades, que exigem maior envolvimento de pessoal das equipes de ambos os parceiros - universidade e empresa (MILES, 2007).

Com base nas conclusões apresentadas, entende-se que uma importante contribuição do artigo é comprovar a relevância dos incentivos governamentais para a cooperação U-E, principalmente em projetos que envolvem maior risco, custo e complexidade e que, por isso, teriam menor comprometimento de recursos privados.

\section{Referências bibliográficas}

ARAUjO, B. C.; PIANTO, D.; DE NEGRI, F.; CAVALCANTE, L. R.; ALVES, P. Impactos dos fundos setoriais nas empresas. Revista Brasileira de Inovação, v. 11, n. esp., p. 85-112, 2012.

ARBIX, G.; CONSONI, F. Inovar para transformar a universidade brasileira. Revista Brasileira de Ciências Sociais, v. 26, n. 77, p. 205-251, 2011.

ARROW, K. Economic Welfare and the allocation of resources for invention. In: MIROWSKI, P.; SENT, E-M. (Ed.). Science bought and sold: essays in the economics of science. Chicago: University of Chicago, 2002.

AVELLAR, A. P.; BOTELHO, M. Políticas de apoio à inovação em pequenas empresas: evidências sobre a experiência brasileira recente. Economia e Sociedade, v. 24, n. 2 (54), p. 379-417, ago. 2015.

BEINHOCKER, E. D. The origin of wealth: evolution, complexity and the radical remaking of economics. US: Random House Business Books, 2007.

CALZOLAIO, A. E.; DATHEIN, R. Políticas fiscais de incentivo à inovação: uma avaliação da Lei do Bem. Porto Alegre: Universidade Federal do Rio Grande do Sul, Faculdade de Ciências Econômicas, 2012 (Texto para discussão, n.15/2012).

CAMPOS, B.; URRACA RUIZ, A. Padrões setoriais de inovação na indústria brasileira. Revista Brasileira de Inovação, v. 8, n. 1, p. 167-210, 2009.

CARRIJO, M.; BOTELHO, M. Cooperação e inovação: uma análise dos resultados do Programa de Apoio à Pesquisa em Empresas (Pappe). Revista Brasileira de Inovação, v. 12, n. 2, p. 417-448, 2013.

CASSIOLATO, J. E.; LASTRES, H. M. Sistemas de inovação e desenvolvimento: as implicações de política. São Paulo em Perspectiva, v. 19, n. 1, p. 34-45, jan./mar. 2005. 
CGGE - Centro de Gestão e Estudos Estratégicos. Programa RHAE - Pesquisador na empresa. Diretório de projetos - Chamadas 67/2008 e 62/2009. Brasília: Centro de Gestão e Estudos Estratégicos, 2011.

COHEN,W.; NELSON, R. R.; WALSH, J. P. The influence of public research on industrial R\&D. Management Science, v. 48, n. 1, p. 1-23, 2002.

FLORIDA R.; COHEN, W. Engine or infrastructure? The university role on economic development. In: BRASCOMBS, L. et al. (Org.). Industrializing knowledge, MIT Press, 1999. p. 589-610.

FREEMAN, C. The "National System of Innovation" in historical perspective. Cambridge Journal of Economics, v. 19, p. 5-24, 1995.

GOLDFARB, B. The effect of government contracting on academic research: does the source the funding affect scientific output? Research Policy, v. 37, p. 41-58, 2008.

GREENE, W. H. Econometric analysis. Prentice Hall: New Jersey, 5th ed., 2002.

GULBRANDSEN, M.; SMEBY, J. Industry-funding and university professor's research performance. Research Policy, v. 34, p. 932-950, 2005.

JENSEN, R.; THURSBY J.; THURSBY, M. C. University-industry spillovers, government funding, and industrial consulting. Cambridge, MA: National Bureau of Economic Research, 2010 (NBER Working Paper, n. 15732).

KANNEBLEY JR., S.; CAROLO, M. D.; DE NGRI, F. Impacto dos fundos setoriais sobre a produtividade acadêmica de cientistas universitários. Estudos Econômicos, v. 43, n. 4, p. 647-685, 2013.

KLEVORICK, A. K.; LEVIN, R.; NELSON, R.; WINTER, S. On the sources and significance of inter-industry differences in technological opportunities. Research Policy, v. 24, n. 2, p. 185205, 1995

LUNDVALL, B. A. National Systems of Innovation: towards a theory of innovation and interactive learning. London: Printer Publishers, 1992.

MANSFIELD, E.; LEE, J. The modern university: contributor to industrial innovation and recipient of industrial P\&D support. Research Policy, v. 25, n. 7, p. 1047-1058, 1996.

MEYER-KRAMER, F.; SCHMOCH, U. Science-based technologies: university-industry interactions in four fields. Research Policy, v. 27, p. 835-851, 1998.

MILES, I. Research and development (R\&D) beyond manufacturing: the strange case of services R\&D. R\&D Management, v. 37, n. 3, p. 249-268, 2007. 
MINGOTI, S. A. Análise de dados através de métodos de estatística multivariada: uma abordagem aplicada. Belo Horizonte: UFMG, 2005.

MOWERY, D. C.; SAMPAT, B. N. Universities in national innovation systems. In: FAGERBERG, J.; MOWERY, D. C.; NELSON, R. R. (Org.) The Oxford handbook of innovation. Oxford: Oxford University Press, 2005.

MUSCIO, A.; QUAGLIONE, D.; VALLANTI, G. Does government funding complement or substitute private research funding to universities. Research Policy, v. 42, p. 63-75, 2013.

NARIN, F.; HAMILTON, K. S.; OLIVASTRO, D. The increasing linkage between U.S. technology and public science. Research Policy, v. 26, n. 3, p. 317-330, 1997.

NELSON, R. Capitalism as an engine of progress. Research Policy, v. 19, p. 193-214, 1990. The simple economics of basic scientific research (1959). In: MIROWSKI, P.; SENT, E-M. (Ed.). Science bought and sold: essays in the economics of science. Chicago: University of Chicago, 2002.

National innovation systems: a comparative analysis. New York: Oxford University, 1993.

PACHECO, C. A. As reformas da politica nacional de ciência, tecnologia e inovação no Brasil (1999-2002). Santiago de Chile: Cepal, dic. 2007.

PEREIRA, N. Fundos setoriais: avaliação das estratégias de implementação e gestão. Brasília: Ipea, 2005 (Texto para discussão, n. 1136).

PERKMANN, M.; WALSH, K. The two faces of collaboration: impacts of university-industry relations on public research. Industrial and Corporate Change, v. 18, n. 6, p.1033-1065, 2009.

RAPINI, M.; OLIVEIRA, V.; SILVA NETO, F. A natureza do financiamento influencia na interação universidade-empresa no Brasil? Revista Brasileira de Inovação, v. 13, n. 1, p. 77-108, 2014.

REDDY, P. The evolving role of university in economic development: the case of universityindustry linkages. In: GORANSON, B.; BRUDENIUS, C. University in transition: the changing role and challenges for academic institutions. Ottawa: Canada: IDRC, 2011.

SCHARTINGER, D.; RAMMER, C.; FISHER, M. M.; FRÖHLICH, J. Knowledge interactions between universities and industry in Austria: sectoral patterns and determinants. Research Policy, v. 31, n. 3, p. 303-328, 2002.

TEIXEIRA, C.; MENEZES, J. H. Resultados do RHAE Pesquisador na Empresa. In: XV CONGRESSO DA ASSOCIAÇÃO LATINO-IBEROAMERICANA DE GESTÃO DE TECNOLOGIA. Anais... Porto, Portugal: Altec, 27 a 31 outubro 2013. 
VAN LOOY, B.; CALLAERT, J.; DEBACKERERE, K. Publications and patent behavior of academic researchers: conflicting, reinforcing or merely co-existing. Research Policy, v. 35, v. 4, p. 596-608, 2004.

VELHO, L.; SAENZ, T. $R \& D$ in the public and private sectors in Brazil: complements or substitutes? Maastricht: The United Nations University, 2002 (UNU/INTECH Discussion Papers Series, 2002-8).

VIOTTI, E. Brasil: de política de C\&T para política de inovação? Evolução e desafios das políticas brasileiras de ciência, tecnologia e inovação. In: VELHO, L.; SOUZA-PAULA, M. C. (Org.). Avaliação de políticas de ciência, tecnologia e inovação: diálogo entre experiências internacionais e brasileiras. Brasília: CGEE, 2008. p. 137-174. 